Trabajo seleccionado para su publicación por el jurado del Premio Estudios Financieros, formado por: don Antonio Bautista Garcia-Vera, don Luis Alberto Guijarro Rojo, doña Ana Hidalgo Tena, doña Blanca Lozano Cutanda y don José Eugenio Martínez Falero.

\title{
¿Es necesario educar en e-health? Conclusiones a partir de las valoraciones de un grupo de estudiantes universitarios
}

\section{Extracto:}

La e-health es un campo de confluencia entre las tecnologías de la informa-

\section{Sumario}

1. Introducción

2. Método

3. Resultados

4. Conclusiones

5. Bibliografía

Anexo ción y la comunicación (TIC) y la salud que está experimentando un importante auge en los últimos años debido a la progresiva incorporación de la sociedad al mundo de las TIC.

El objetivo de este trabajo es averiguar cuáles son las actitudes y los comportamientos de un grupo de universitarios respecto a la e-health. Para ello, se realizó una encuesta sobre el uso de diferentes aspectos de la e-health a 360 estudiantes de grado y posgrado de la UDIMA, de los cuales el 67,5\% eran mujeres y el $32,5 \%$ hombres. La edad media de la muestra fue 37,2 años (DT: 9,55).

Los resultados del estudio muestran un uso importante de internet como fuente de información sobre salud, así como de otras funcionalidades de la e-health. No obstante, los participantes también manifestaron cierto desconocimiento y desconfianza en relación a las posibilidades que este nuevo campo ofrece.

Palabras clave: competencias digitales, e-health, salud, tecnologías de la información y la comunicación (TIC).

Fecha de entrada: 03-05-2016

Fecha de aceptación: 12-07-2016

\footnotetext{
J. I. Baile Ayensa, vicerrector de ordenación académica y profesor del Departamento de Psicología de la Facultad de Ciencias de la Salud y la Educación de la UDIMA.

${ }^{2}$ M. a J. González-Calderón, profesora del Departamento de Psicología de la Facultad de Ciencias de la Salud y la Educación de la Universidad a Distancia de Madrid (UDIMA).

${ }^{3}$ R. Palomo Santos, profesora del Departamento de Psicología de la Facultad de Ciencias de la Salud y la Educación de la Universidad a Distancia de Madrid (UDIMA).
} 


\section{Is it necessary to educate on e-health?} Conclusions based on the evaluation of a group of university

\section{Abstract:}

E-health is a new field in which the information and communication technologies (ICT) and the health knowledge converge. It has undergone a significant growth in the last years due to the continual incorporation of citizens to the world of ICT.

The aim of this study is to find out what the attitudes and behaviors of a group of university students are regarding e-health. 360 university students taking a bachelor's or a master's degree at UDIMA university filled in a survey on the use of different aspects of e-health. The sample's mean age was 37.2 years old (SD: 9.55 ), $67.5 \%$ of the subjects were women and $32.5 \%$ were men.

A significant use of the internet and other e-health tools as a source of information on health was found. However, participants also expressed certain suspicion and lack of awareness in relation to the possibilities this new field may offer.

Keywords: digital competence, e-health, health, information and communication technologies (ICT).

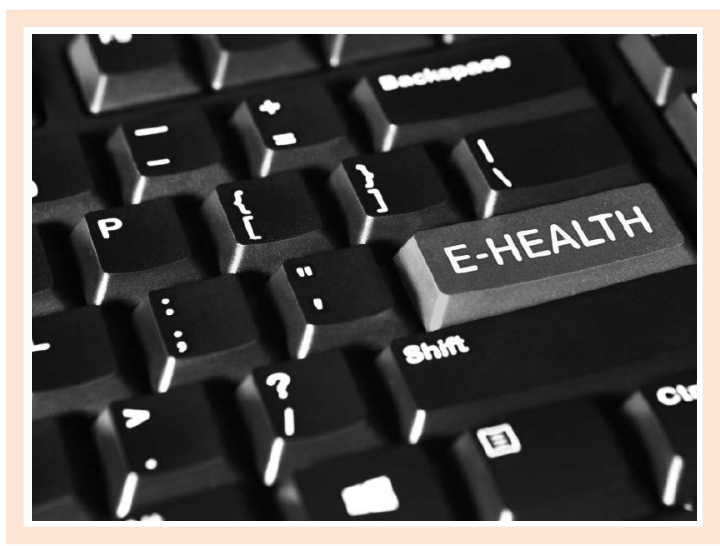

\section{INTRODUCCIÓN}

En la era de las nuevas TIC, prácticamente todos los ámbitos del ser humano están influidos por ellas, tal y como se observa en el contexto lúdico 0 de entretenimiento, en el de la enseñanza-aprendizaje, en el laboral, en el relativo a las relaciones personales y, por supuesto, en el vinculado a la salud. De hecho, en la actualidad, en los países desarrollados, el cuidado de la salud es uno de los ámbitos donde las TIC ofrecen un amplio abanico de opciones tanto a los usuarios como a los profesionales sanitarios. El desarrollo en este campo es tal, que ya tiene una denominación propia: «e-salud», «cibersalud» 0 «e-health». Este último término inglés, que posee una gran difusión, será el que se emplee en el presente estudio para referirnos al empleo de las TIC en el ámbito de la salud.

Se han propuesto diferentes definiciones de e-health desde distintos paradigmas, como indican Oh et al. (2005). La Organización Mundial de la Salud (OMS) define este concepto como «el uso rentable y seguro de las TIC en apoyo de la salud y ámbitos relacionados con ella, que incluye los servicios de atención sanitaria, la vigilancia de la salud, la bibliografía existente sobre ella, así como la educación, los conocimientos e investigaciones en materia de salud» (OMS, 2005).

Una de las definiciones mencionadas con más frecuencia es la propuesta por Eysenbach (2001), para quien el concepto de e-health describe «un campo emergente en la intersección de la informática médica, la salud pública y los negocios, en referencia a los servicios de salud y la información obtenida o tratada a través de internet y las tecnologías relacionadas». Este autor 
considera, además, que, en un sentido más amplio, «el término representa no solo un desarrollo técnico, sino también una nueva mentalidad, una manera de pensar, una actitud y un compromiso con un pensamiento en red, global, para mejorar la atención de la salud a nivel local, regional y en todo el mundo mediante el uso de las TIC». Desde esta visión amplia y comprehensiva, la e-health se considera no como un simple conjunto de herramientas y servicios de las TIC aplicados a la salud, sino también como un nuevo paradigma en esta área. Probablemente la forma en la que actualmente entendemos la salud (desde una perspectiva informativa, educativa, preventiva, de diagnóstico o de tratamiento, etc.) cambiará radicalmente gracias a la e-health. De ahí nace la importancia de conocer hasta qué punto la población es consciente de ello y cómo va adaptándose a esta nueva realidad.

Como puede apreciarse, la e-health permite un nuevo formato de intervención sanitaria que incluye aspectos preventivos y formativos, y que llega a nuevos actores antes inaccesibles. En la actualidad, está organizada en torno a una gran diversidad de servicios y productos, como son las páginas web con información acerca de la salud, los dispositivos de monitorización ${ }^{4}$ (como los pulsímetros), las aplicaciones para los teléfonos móviles, la telemedicina, el uso del big data ${ }^{5}$, los videojuegos relacionados con la salud o los sistemas de comunicación entre el profesional de la salud y el usuario (teléfono, e-mail, aplicaciones de mensajería, etc.). También se está convirtiendo en un campo de gran desarrollo desde el punto de vista económico, pues se estima que a finales de 2015 el mercado global de e-health había alcanzado los 160.000 millones de dólares, con una tasa de crecimiento anual compuesto para el periodo 2010-2015 del 16\% (Fundación Telefónica, 2016).

\footnotetext{
${ }^{4}$ Es muy común usar el término inglés wearable (procedente del verbo inglés to wear [llevar puesto]) para referirse a este tipo de dispositivos o aparatos electrónicos que se incorporan en alguna parte de nuestro cuerpo, interactuando continuamente con el usuario y con otros dispositivos con la finalidad de realizar alguna función específica, como las pulseras que monitorizan nuestro estado de salud.

${ }^{5}$ Expresión inglesa que se refiere al manejo de gran cantidad de datos para el estudio del comportamiento de las personas con el objetivo de, en la medida de lo posible, predecirlo. Un ejemplo de big data lo constituiría el estudio de la prevalencia de una enfermedad estacional mediante el análisis de las búsquedas de información que en internet se llevan a cabo en relación a los síntomas o tratamientos asociados a dicha enfermedad.
}

Si bien, en algunas de sus manifestaciones, la ehealth se viene utilizando durante las dos últimas décadas, el conocimiento acerca de su implantación, el uso que hacen de ella las personas y el grado de confianza, etc., es bastante desconocido. Esto puede llevar a una posible infrautilización de las posibilidades que ofrece en la atención de un aspecto tan importante para los ciudadanos como es la salud. Téngase en cuenta, por ejemplo, que según el Instituto Nacional de Estadística (INE), en el $96,4 \%$ de los hogares españoles hay al menos un smartphone (Morales, 2015), lo cual permitiría a la práctica totalidad de los habitantes el acceso a gran parte de las herramientas empleadas desde la e-health: la telemedicina, el e-learning, la m-health, etc. No obstante, y a pesar de la disponibilidad o accesibilidad técnica mencionada, se plantea la siguiente duda: ¿realmente se valoran y se emplean eficazmente dichas herramientas?

Las cuestiones que se plantean en este ámbito, y que han motivado la presente investigación, se resumen en los siguientes aspectos: ¿conoce la población española el campo de la e-health? y ¿utiliza las herramientas disponibles al respecto?

Con objeto de resolver las cuestiones previamente señaladas, se plantea el siguiente objetivo de investigación: estudiar los comportamientos y las actitudes de un grupo de estudiantes universitarios españoles respecto al uso de las TIC en el ámbito de la salud para determinar si es necesario mejorar la educación sobre e-health.

Una de las definiciones mencionadas
con más frecuencia es la propuesta
por Eysenbach (2001), para quien el
concepto de e-health describe «un
campo emergente en la intersección
de la informática médica, la
salud pública y los negocios, en
referencia a los servicios de salud y
la información obtenida o tratada a
través de internet y las tecnologías
relacionadas»

Una de las definiciones mencionadas con más frecuencia es la propuesta por Eysenbach (2001), para quien el concepto de $e$-health describe un campo emergente en la intersección de la informática médica, la salud pública y los negocios, en referencia a los servicios de salud y la información obtenida o tratada a relacionadas» 
Como objetivos específicos de este estudio, se presentan los que enumeramos a continuación:

- Averiguar si la muestra estudiada hace uso de las TIC para controlar algún aspecto de su salud.

- Conocer qué tipo de TIC utiliza la muestra seleccionada para controlar u obtener información respecto a la salud.

\section{MÉTODO}

\subsection{Participantes}

La muestra fue seleccionada de manera incidental. Estuvo compuesta por 360 estudiantes de la UDIMA con edades comprendidas entre los 20 y los 60 años (media: 37,32; DT: 9,55). De ellos, el 67,5\% eran mujeres y el $32,5 \%$ hombres. Las características descriptivas de la muestra pueden observarse en la tabla 1.

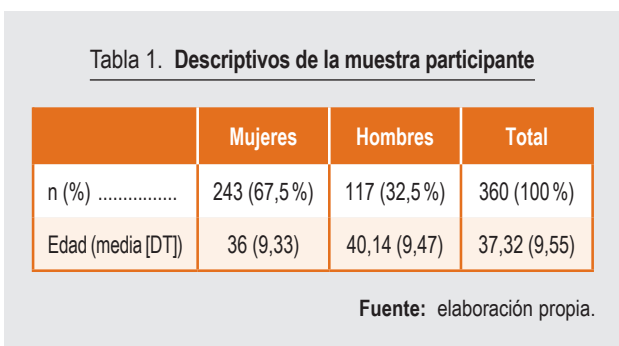

Respecto a la formación previa de los participantes, independientemente de la titulación universitaria que cursaban, se observó que el $60,9 \%$ disponía de estudios universitarios previos (licenciatura, diplomatura, grado, máster o doctorado), el 15,8\% contaba con formación secundaria (bachillerato), el 10\% poseía otra titulación (formación profesional, titulaciones extranjeras, etc.) y el 13,3\% había accedido a los estudios universitarios a través de pruebas de acceso para mayores de 25, 40045 años. La alta proporción de estudiantes con títulos universitarios previos se debió a que parte de la muestra procedía de estudiantes de másteres oficiales que requieren, obligatoriamente, disponer de un título universitario para acceder a ellos. Por otro lado, por el perfil e idiosincrasia del alumnado de esta universidad, es habitual que los estudiantes cuenten con estudios previos. Por tanto, el nivel educativo de la muestra fue, en términos generales, alto.
- Analizar cuál es el grado de confianza que la muestra tiene en relación al empleo de las TIC en aspectos relacionados con la salud.

- Indagar sobre cuáles son las razones para utilizar las TIC en asuntos relacionados con la salud.

- Analizar si existen diferencias respecto a los aspectos previos en función del género de los participantes.

\subsection{Instrumento de evaluación}

Para llevar a cabo la encuesta acerca de la experiencia de los participantes y su valoración respecto a los diversos aspectos de la e-health se elaboró un cuestionario ad hoc, el cual se puede ver en el anexo situado al final de este estudio. El citado cuestionario constaba de 25 preguntas que recopilaban tanto variables sociodemográficas (género, edad, nivel educativo), como aspectos referidos al objeto de la investigación. Dichas cuestiones se podían contestar a través de opciones excluyentes o de casillas de verificación en las que era posible seleccionar más de una respuesta. Para su elaboración se consultaron estudios previos sobre temática similar, como el llevado a cabo por el Observatorio Nacional de las Telecomunicaciones y de la Sociedad de la Información (ONTSI, 2012).

\subsection{Procedimiento}

Los estudiantes fueron invitados a participar en la investigación de forma anónima y voluntaria, solicitándoles que respondieran a una encuesta mediante la herramienta Formulario de Google Drive. Dicha invitación y el link al formulario lo recibieron a través de los tablones de las aulas centrales de las titulaciones correspondientes mediante un mensaje de los respectivos decanos de las facultades implicadas (Facultad de Ciencias de la Salud y la Educación y Facultad de Ciencias Jurídicas). Los estudiantes dispusieron de una semana para decidir si

\section{El nivel educativo de la muestra fue, en términos generales, alto}


cumplimentaban la mecionada encuesta, que no se tardaba más de tres minutos en contestar.

Una vez finalizado el plazo para la realización de la encuesta y recopilados los datos, estos fueron analizados mediante el programa informático Excel y el programa estadístico online WinEpi.

\subsection{Diseño}

En esta investigación se llevó a cabo un estudio transversal mediante la metodología de encuesta.

\section{RESULTADOS}

A continuación, se indican los principales resultados obtenidos en la investigación. Estos se han agrupado por ámbitos temáticos, respondiendo a los objetivos del estudio.

\subsection{Empleo de las TIC en el ámbito de la e-health}

\subsubsection{Dispositivos empleados a la hora de realizar consultas en internet}

A los participantes no se les consultó si accedían o no a internet, pues la muestra estaba compuesta por estudiantes que cursaban sus estudios en una universidad online. Asimismo, el instrumento de recogida de datos consistió en un formulario online que los participantes recibieron en su correo electrónico, por

\section{Los estudiantes fueron invitados a participar en la investigación de forma anónima y voluntaria, solicitándoles que respondieran a una encuesta mediante la herramienta Formulario de Google Drive}

lo cual era evidente que el $100 \%$ de los participantes de la muestra tenía acceso a internet.

Con respecto a qué dispositivos informáticos utilizaban los participantes habitualmente, el $90,1 \%$ de los encuestados señalaron que empleaban el teléfono móvil; el $87 \%$, el ordenador portátil; el 45,6\%, el ordenador de sobremesa; y el 49,4\%, la tableta. Por tanto, se observa que son los dispositivos móviles (teléfono u ordenador con estas características) los más empleados, ya que solo en torno al $10 \%$ de la muestra no los usaba habitualmente.

Si indagamos sobre cuál de los dispositivos se utilizaba para realizar las consultas en internet, comprobamos que el ordenador o las tabletas eran los preferidos frente al teléfono móvil, si bien este también era empleado en un porcentaje elevado (véase figura 1).

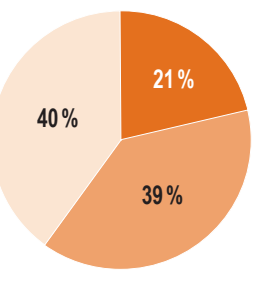

Principalmente el teléfono móvil

Principalmente el ordenador o la tableta

Uso por igual el móvil y el ordenador o la tableta 


\subsubsection{Realización de búsquedas de información acerca de la salud en internet: frecuencia de las mismas, estrategias empleadas para llevarlas a cabo y comparación con otras fuentes de información sobre salud}

Cuando se les preguntó a los participantes si buscaban información sobre salud en internet (enfermedades, tratamientos, síntomas, profesionales de la salud, etcétera), una abrumadora mayoría afirmaron que si lo hacían $(80,9 \%)$, frente al $19,1 \%$ que indicó que no realizaba ese tipo de búsquedas en internet.

En relación a la frecuencia con la que se buscaba información sobre salud en internet, los resultados indicaron que se realizaban este tipo de búsquedas con bastante asiduidad, dado que el $37 \%$ de los participantes de la muestra llevaba a cabo estas consultas varias veces al mes o con mayor frecuencia (véase figura 2). También es interesante comprobar que solo el $16,1 \%$ de la muestra señaló que nunca llevaba a cabo búsquedas de este tipo.

Figura 2. Frecuencia con la que los participantes realizan consultas sobre salud en internet

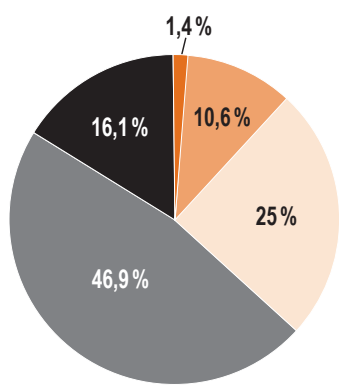

A diario

Varias veces a la semana

Varias veces al mes

Una vez al mes o con menor frecuencia

Nunca realizo ese tipo de búsquedas
Respecto de las estrategias empleadas por la muestra en la búsqueda de información sobre salud en la red, como puede observarse en la figura 3 , los encuestados respondieron que utilizaban de forma abrumadora buscadores clásicos como Google $(78,6 \%)$. Por otro lado, es significativo que solo 1 de cada 5 participantes consultara páginas específicas de salud.

En relación al uso de otras fuentes de información sobre la salud distintas de las proporcionadas desde la ehealth (diccionarios médicos, libros o revistas médicas o especializadas en salud u otras), en términos generales, se observó un uso muy reducido de estas fuentes (véase figura 4).

Figura 3. Estrategias empleadas por la muestra en su búsqueda de información sobre salud en internet

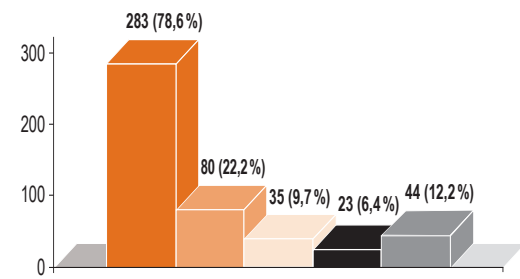

Buscadores (Google)

Páginas web de salud

Redes sociales

Otras fuentes de internet

Ninguno

Fuente: elaboración propia. 


\section{Figura 4. Empleo de otras fuentes de información «tradicionales» (en papel) sobre salud distintas de la e-health}

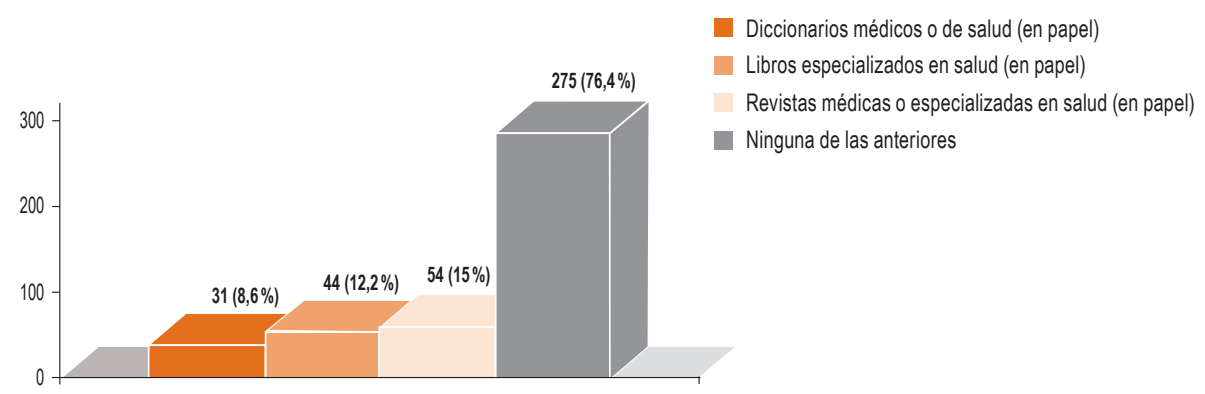

Fuente: elaboración propia

\subsubsection{Aplicaciones de control y/o seguimiento de aspectos relacionados con la salud}

El acceso a la e-health puede realizarse por diferentes medios, más allá de las búsquedas de información en internet. Acerca de este aspecto, también se preguntó a la muestra por el control y el seguimiento de su actividad física, observándose que casi 4 de cada 10 sujetos utilizaban algún dispositivo de monitorización de dicha actividad y/o aplicación del móvil para el control o el seguimiento de la misma, siendo esta última la más común $(27,3 \%)$. Otro tipo de programas informáticos o páginas web específicas para lograr el mismo objetivo en relación a la actividad física parecen tener un seguimiento anecdótico. En la figura 5 se recoge la información más importante al respecto.

Figura 5. Empleo de dispositivos o aplicaciones de control y/o seguimiento de la actividad física

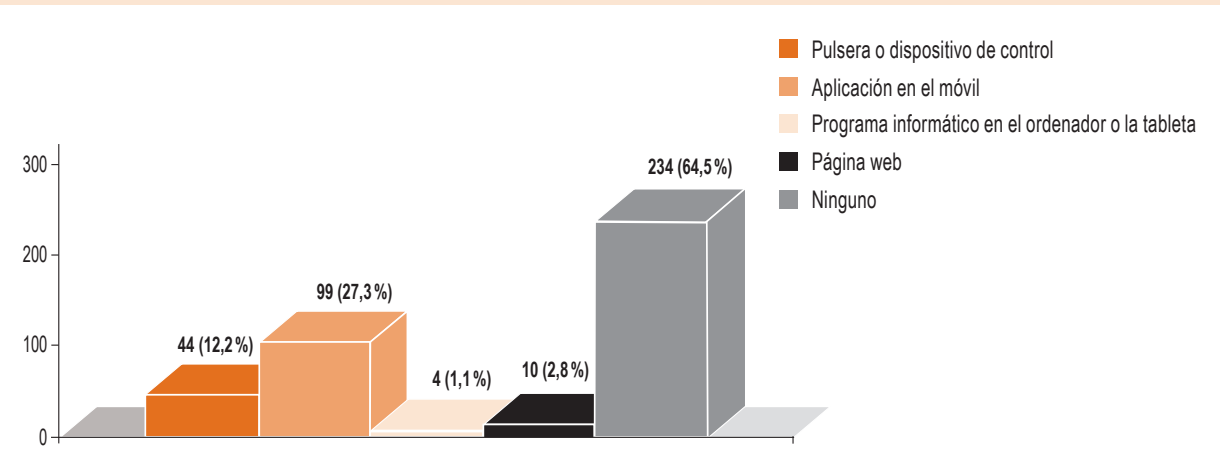

Fuente: elaboración propia. 


\section{2. ¿Cuál suele ser el motivo para emplear la e-health?}

\subsubsection{Objetivo de las aplicaciones (app) de salud empleadas}

En referencia al uso de las aplicaciones para el control de la actividad física, la alimentación u otras variables relacionadas con la salud, los resultados mostraron que, si bien todavía tienen una implantación limitada (el $65,6 \%$ aún no las utiliza), ya se aprecia un número importante de sujetos que las usan, pues aproximadamente uno de cada cuatro encuestados universitarios las empleaban, siendo el objetivo más común el seguimiento y el control de la actividad física $(21,2 \%)$. En la figura 6 se presentan los resultados al respecto.

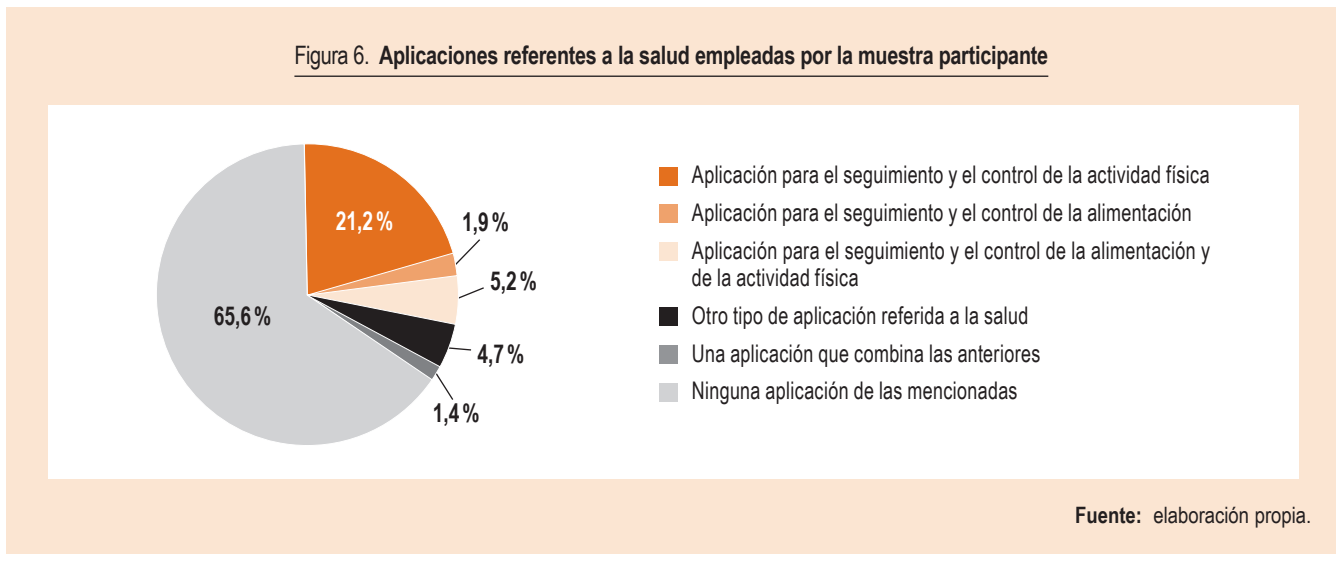

\subsubsection{Motivos para consultar información sobre temas de salud en internet}

Si analizamos el motivo que tenían los participantes para consultar información sobre temas de salud en internet, puede concluirse que la razón principal era resolver dudas de salud $(36,9 \%)$, así como obtener mayor información sobre los tratamientos vinculados a un problema determinado de salud (35,8\%). En la figura 7 pueden observarse las respuestas de los participantes acerca de esta cuestión.

\section{Figura 7. Motivos de los participantes para realizar consultas de salud en internet}

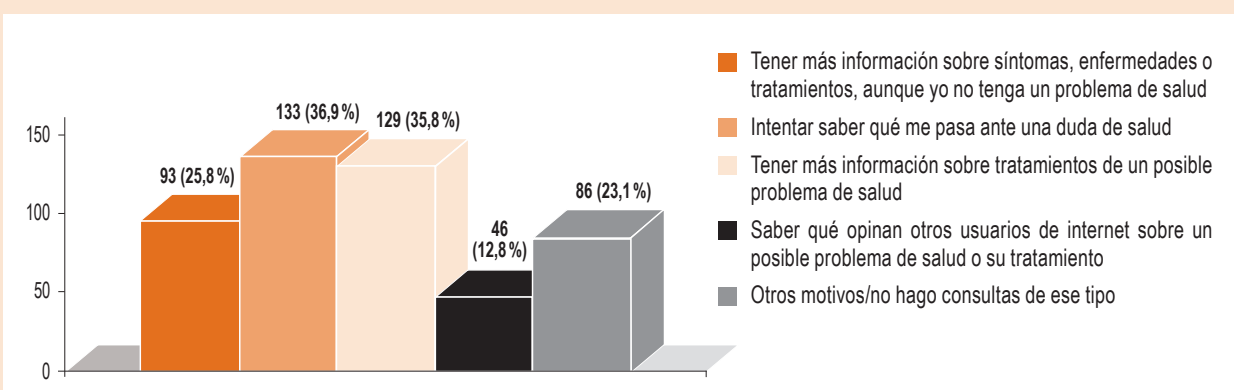

Fuente: elaboración propia. 


\subsubsection{Efectos de las búsquedas de informa- ción sobre temas de salud en internet}

Respecto a la pregunta de si habían seguido algún tratamiento relacionado con un problema de salud a partir de la información obtenida en internet, el 88,9\% de los encuestados contestaron negativamente, frente a un $11,1 \%$ que indicaron que sí lo habían seguido. Por otro lado, gran parte de la muestra $(97 \%)$ indicó que no habían comenzado un tratamiento farmacológico, ni habían modificado uno ya recetado por un profesional médico en su consulta, a partir de la información obtenida en internet.

\subsubsection{Consultas a profesionales de la salud a través de internet}

En relación a la cuestión de si los participantes habían consultado a profesionales de la salud a través de internet (psicólogos, nutricionistas, pediatras, ginecólogos, etc.), la mayoría contestaron negativamente $(88,7 \%)$, frente al $11,3 \%$ que señalaron que sí lo habían hecho. A aquellos que contestaron que nunca habían consultado a través de este medio, se les preguntó si lo harían en el futuro; de ellos, el 36,9\% afirmaron que sí lo harían. Por tanto, puede concluirse que casi la mitad de la muestra, o bien había consultado a algún profesional de la salud a través de internet, o bien estaba dispuesto a hacerlo.

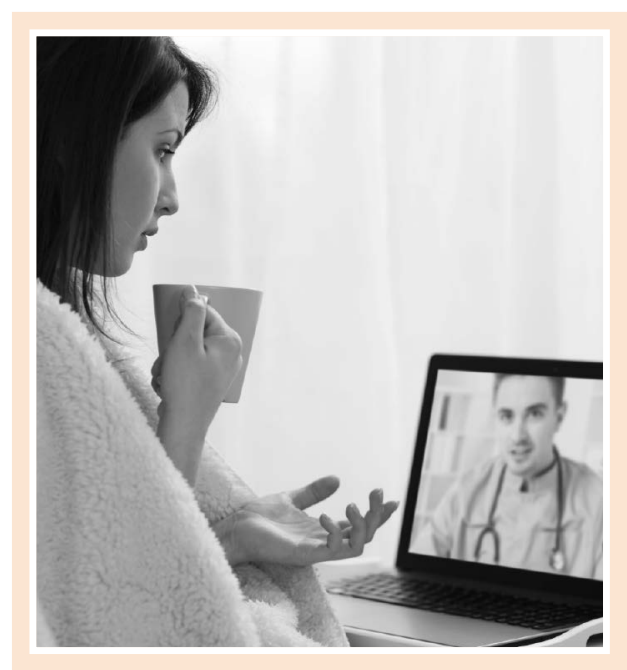

\subsection{Grado de confianza en la e-health}

\subsubsection{Grado de confianza en la información sobre salud}

Cuando a los participantes del estudio se les cuestionó sobre qué grado de confianza tenían en la información que se podía conseguir a través de internet acerca de la salud, se comprobó que solo el 1,9\% de los participantes afirmaba que les proporcionaba una total confianza, si bien más de la mitad $(57 \%)$ consideraban que este tipo de información les generaba un nivel medio de confianza. La puntuación media obtenida por la muestra (2,93 sobre 5 [DT: 0,82]) sirve de indicador de la valoración de la confianza. En la figura 8 pueden observarse las valoraciones de la muestra al respecto.

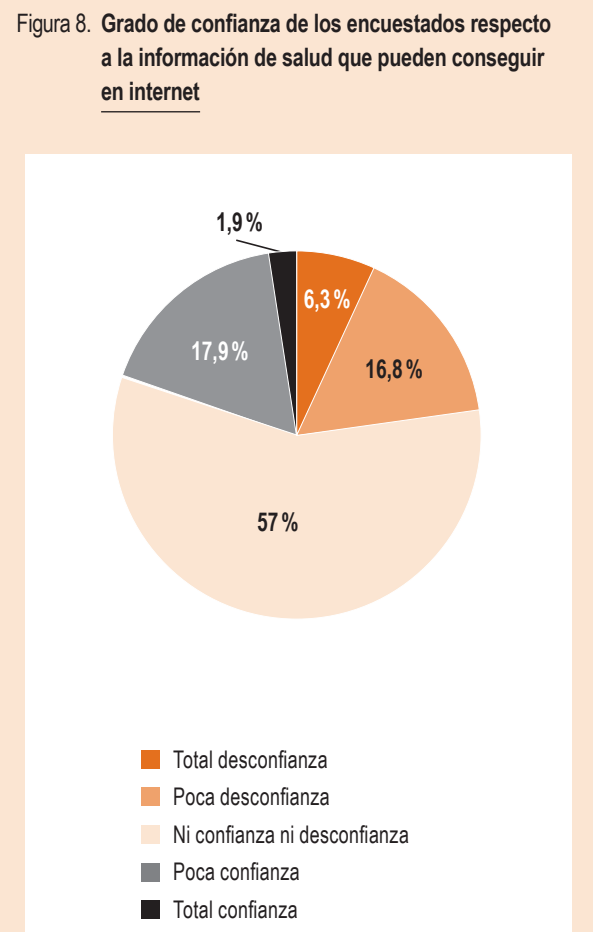

Fuente: elaboración propia 


\subsubsection{Grado de confianza en un servicio de salud proporcionado a través de internet y motivos para no emplearlo}

En relación al grado de confianza que suscitaba en los participantes de la encuesta recibir un servicio de salud a través de internet (por ejemplo, algún tipo de terapia vía online), los resultados revelaron que casi la mitad de ellos $(52,8 \%)$ demostraba poca o muy poca confianza al respecto. Por otro lado, es también destacable que únicamente el $0,5 \%$ de los participantes mostraban total confianza en esta nueva modalidad sanitaria (véase figura 9). Quizás todos estos datos explican por qué razón solo un $3,6 \%$ de los en- cuestados había recibido tratamiento online por parte de un profesional de la salud.

En relación a cuáles serían los motivos para no emplear servicios de salud online, las respuestas más contundentes giraron en torno a la desconfianza: por una parte, el $60 \%$ de la muestra desconfiaba de la identidad del profesional; asimismo, un 47,8\% lo hacía de su profesionalidad. En la figura 10 se presentan las respuestas de los participantes sobre esta cuestión.

\section{Figura 9. Grado de confianza de los encuestados respecto a recibir un servicio de salud a través de internet}

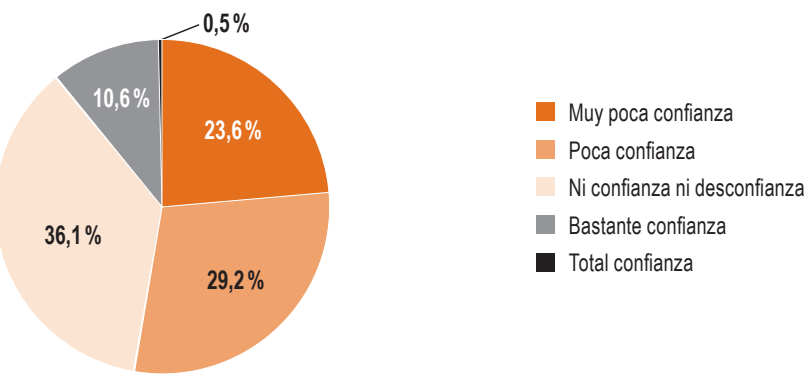

Fuente: elaboración propia.

\section{Figura 10. Motivos para no emplear servicios de salud online}

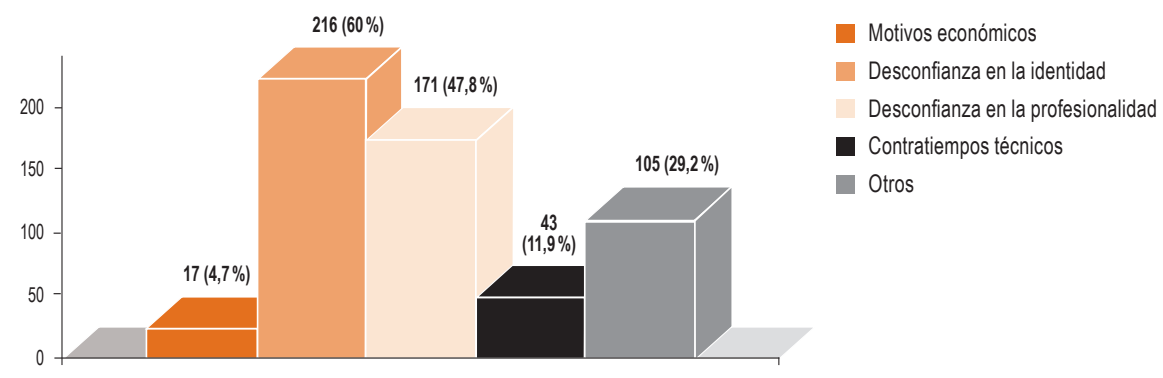

Fuente: elaboración propia. 


\subsection{Valoración comparativa acerca de las consultas a profesionales de la salud a través de internet versus presenciales}

\subsubsection{Relación entre las consultas de salud online y las presenciales}

En este estudio se indagó acerca de la relación entre la e-health y las consultas físicas o presenciales a profesionales de la salud. Así, se les preguntó a los participantes sobre posibles acciones de e-health que pudieran llevar a cabo cuando acudiesen presencialmente a consulta por temas de salud (al médico de cabecera, al especialista, al psicólogo, al enfermero, etc.). En concreto, se les cuestionaba sobre si buscaban información en internet sobre lo que les pasaba antes de la consulta presencial, después de esta o en ambas ocasiones (antes y después). Los resultados al respecto son muy contundentes, ya que el $63,9 \%$ de los participantes señalaba que buscaba información antes y/o después de la consulta presencial, frente al $36,1 \%$ que indicaba no hacerlo nunca. En la figura 11 se observan las respuestas de los participantes al respecto.

\subsubsection{Valoración de las consultas online ver- sus presenciales}

Profundizando en la confianza de los participantes en la e-health, también se les pidió que compararan la atención sanitaria proporcionada online con los servicios presenciales (cara a cara), indicando si consideraban que el servicio de salud presencial era mejor, igual o peor que el proporcionado por un profesional sanitario en la red.

De forma rotunda la muestra señaló que el servicio presencial era mejor (87,5\%), si bien 1 de cada 10 participantes estimaba que era igual (véase figura 12).

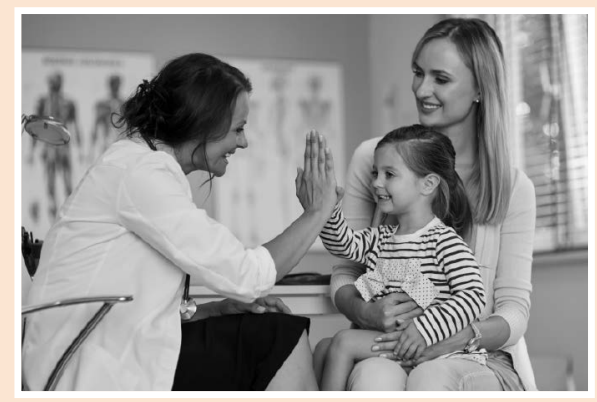

Figura 11. Búsqueda de información sobre salud en internet relacionada con consultas de salud presenciales por parte de la muestra

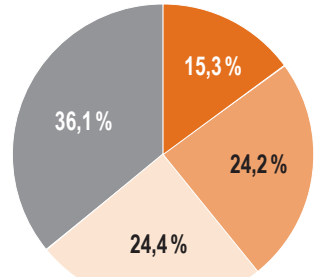

Antes de ir a consulta, consulto información en internet

Después de la consulta, consulto información en internet

Suelo consultar información antes y después de la consulta

No suelo consultar información en internet ni antes ni después de la consulta

Fuente: elaboración propia.

Figura 12. Valoración de los servicios de salud online versus presenciales

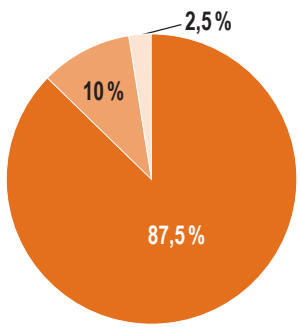

Considero el servicio presencial mejor que el servicio online

Considero el servicio presencial igual que el servicio online

Considero el servicio presencial peor que el servicio online

Fuente: elaboración propia 


\subsection{Diferencias de género en el ámbito de la e-health}

Con objeto de indagar sobre las diferencias que hombres y mujeres presentan en relación a sus comportamientos y actitudes frente a la e-health, se llevaron a cabo análisis estadísticos para comparar las tendencias de respuesta en ambos géneros en aquellos ítems que se consideraron de mayor interés. En la tabla 2 pueden observarse los resultados de este análisis.

La única diferencia estadísticamente significativa que se observó en función del género de los participantes es la relativa a la realización de búsquedas de información acerca de salud en internet, siendo las mujeres las que las llevan a cabo en mayor proporción que los varones, quizás debido a que suelen estar más implicadas en la crianza de los hijos y en el cuidado de los ancianos, poblaciones sobre las que se realizan gran parte de las consultas de salud, tanto presencial como virtualmente (online). Sin embargo, exceptuando este aspecto, no se observan diferencias por género en el resto de actitudes y comportamientos referidos a la e-health analizados (grado de confianza que les ofrece la información sobre e-health que pueden obtener en las redes; seguimiento de tratamientos relacionados con un problema de salud a partir de la información obtenida en internet; inicio de tratamientos farmacológicos o modificación de un tratamiento recetado por un profesional médico a partir de la información obtenida en internet; tendencia a consultar información sobre salud en internet antes o después de una visita presencial a un profesional de la salud, etc.).

\section{Tabla 2. Diferencias en el empleo de la e-health en función del género}

\begin{tabular}{|c|c|c|c|c|c|}
\hline & Muestra total & Mujeres & Hombres & $\begin{array}{c}X^{2} \\
\text { (chi-cuadrados) }\end{array}$ & $\mathbf{P}$ \\
\hline 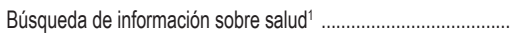 & $80,9 \%$ & $86,8 \%$ & $62,4 \%$ & 28.214 & $<0.0001$ \\
\hline Grado de confianza en información sobre salud en internet ${ }^{2}$..... & $19,7 \%$ & $17,7 \%$ & $24 \%$ & 1.978 & 0.1596 \\
\hline 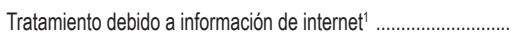 & $13,7 \%$ & $14,8 \%$ & $12 \%$ & 0.518 & 0.4718 \\
\hline $\begin{array}{l}\text { Iniciar/modificar tratamiento farmacológico a partir de } \\
\text { información de internet }{ }^{3}\end{array}$ & $97,1 \%$ & $97,1 \%$ & $97,4 \%$ & 0.027 & 0.86 \\
\hline $\begin{array}{l}\text { Búsqueda de información en internet antes/después de una } \\
\text { consulta presencial' }\end{array}$ & $63,9 \%$ & $66,2 \%$ & $58,1 \%$ & 2.237 & 0.1347 \\
\hline
\end{tabular}

\footnotetext{
Nota. En los análisis estadísticos se ha considerado un nivel de confianza de 0.950 y g.l. $=1$

1 Se han analizado las respuestas afirmativas.

2 Se han analizado las respuestas de alta o total confianza.

3 Se han analizado las respuestas negativas.
}

\section{CONCLUSIONES}

Los participantes de este estudio de investigación, con un nivel educativo que puede considerarse relativamente elevado, y que están acostumbrados al empleo de las TIC, al menos en el ámbito de la enseñanzaaprendizaje, al cursar sus estudios superiores en la modalidad online, muestran las siguientes tendencias en relación a la e-health:
- A pesar del perfil previamente mencionado, aproximadamente 3 de cada 5 encuestados o nunca ha realizado consultas sobre salud en internet o lo hace con una frecuencia igual o inferior a «alguna vez al mes»; asimismo, solo 1 de cada 5 realiza dichas consultas mediante páginas especializadas en salud. Por el contrario, la mayoría emplean los tí- 


\section{[...] la muestra participante realiza en un porcentaje elevado consultas en internet fundamentalmente cuando quiere ampliar información acerca de enfermedades o síntomas que padece}

picos buscadores que pueden llevarles a páginas carentes de todo rigor científico-médico. Lo que sí parece que se constata es la falta de empleo de los recursos en papel en la actualidad (diccionarios, libros o revistas especializadas) a la hora de realizar consultas sobre salud, ya que estos son empleados únicamente por alrededor del $10 \%$ de la muestra.

- Esta muestra vinculada al mundo de las TIC tampoco emplea en una proporción significativa aquellas aplicaciones asociadas a la salud; de hecho, la cifra no alcanza a 2 de cada 5 participantes. Dentro del ámbito de la salud, las aplicaciones empleadas en mayor medida son aquellas vinculadas al seguimiento y al control de la actividad física. Es interesante comentar que quienes las emplean se decantan por las pulseras o por las aplicaciones para el móvil, frente a otras herramientas de e-health.

- Es destacable el hecho de que la muestra participante realiza en un porcentaje elevado consultas en internet fundamentalmente cuando quiere ampliar información acerca de enfermedades o síntomas que padece y solo 1 de cada 10 encuestados está interesado en conocer opiniones de otros internautas al respecto. Otro dato positivo es el hecho de que solo 1 de cada 10 participantes dice haber llevado a cabo tratamientos en función de la información encontrada en la red; asimismo, apenas ningún encuestado afirma haber modificado las pautas aconsejadas previamente por un profesional de la salud.

- Los momentos que parecen llevar a las personas a consultar información sobre salud son aquellos próximos en el tiempo a las visitas a profesionales de la salud.

- A pesar de la idiosincrasia de la muestra, tampoco hay apenas participantes que hayan consulta- do a profesionales de la salud en la red (aproximadamente 1 de cada 10), lo que contrasta con el dato de que casi 4 de cada 10 de los que aún no lo han hecho afirman que lo harían. Estrechamente relacionado con este aspecto, es muy revelador el dato relacionado con el grado de confianza de la muestra en la e-health. Los datos reflejan que los ciudadanos aún no saben qué opinar en relación a la información que pueden obtener en la red, encontrándose la mayoría de las respuestas en el valor intermedio (ni confían ni desconfían) y no habiendo apenas valores extremos en esta variable. Sin embargo, cuando la pregunta se centra en la confianza en un servicio de salud no meramente informativo, los niveles de confianza se reducen, $y$, aunque parte de la muestra también refleja valores intermedios, más de la mitad de los participantes presentan una alta o total desconfianza al respecto. Esto parece deberse a la desconfianza en el profesional de la salud que pudiera prestarle sus servicios, ya que parecen dudar acerca de que este sea quien dice ser, así como de que realmente posea las competencias requeridas para desempeñar su labor. Quizá esto explica por qué solo el $3 \%$ de los encuestados considera que el servicio de salud presencial es peor que el realizado online, decantándose en su mayoría por el primero.

- Por último, es necesario apuntar que apenas se han hallado diferencias reseñables en función del género de los participantes en relación a la e-health. Únicamente las mujeres parecen buscar información sobre salud en la red en mayor proporción que los varones, probablemente debido a los argumentos previamente mencionados acerca de su rol de cuidadoras, especialmente de niños y ancianos en el ámbito doméstico.

Si comparamos los hallazgos obtenidos entre este estudio y otros que han explorado las actitudes de los ciudadanos hacia la e-health, por ejemplo, con la investigación del ONTSI (2012), que empleó población general, encontramos estas singularidades:

- En relación al empleo de internet como fuente de información sobre salud, dicho estudio detectó que un $48,3 \%$ de los participantes lo utilizaban. En el presente estudio la cifra obtenida es claramente superior $(80,9 \%)$. Ello puede ser debido a dos razones fundamentales: por un lado, a la singulari- 
dad de la muestra (estudiantes universitarios, con alto nivel educativo, y que además cursan estudios superiores online); por otro lado, el hecho de que el estudio del ONTSI se llevó a cabo hace cuatro años, y en este periodo de tiempo probablemente los valores se habrán incrementado notablemente, incluso en población general, debido al progresivo aumento del uso de las TIC en todos los ámbitos.

- Respecto a la frecuencia de uso de internet para la búsqueda de información sobre salud, los resultados obtenidos en el presente estudio se asemejan a otros previos. Por ejemplo, el $29,8 \%$ de los internautas del estudio ONTSI había realizado consultas durante la última semana, dato comparable al $37 \%$ de la presente muestra, que indicaba que realizaban consultas al menos varias veces al mes.

- Si se analiza el objetivo de la búsqueda de información en la red, en ambos estudios se obtienen valores similares, siendo el primer motivo obtener información relativa a síntomas, enfermedades y dudas sobre salud, si bien en el presente trabajo las preguntas eran algo más específicas.

- Con respecto a la confianza que ofrece internet en el ámbito de la salud, en nuestro estudio se obtuvo un resultado promedio de 2,9 sobre 5. En el llevado a cabo por la ONTSI se halló un valor algo inferior entre los internautas $(4,4$ sobre 10$)$ y bastante más bajo entre los no usuarios de internet $(2,46$ sobre 10). De nuevo, la existencia de una mayor confianza en internet como fuente de salud en la presente muestra puede ser debida a las dos razones expuestas en el primer punto de este análisis comparativo, así como a la singular composición de la muestra, pues parte de ella está formada por estudiantes de la Facultad de Ciencias de la Salud y la Educación de la UDIMA, quienes se espera que sean capaces de realizar búsquedas de información en internet en el campo de la salud de manera más fiable que otros individuos.

- Respecto a las diferencias de género, los resultados obtenidos en el presente estudio también son comparables con los de la ONTSI, pues en ambos solo se encontró que las mujeres utilizaban internet para buscar información sobre salud en mayor medida que los hombres.

- Otro dato comparable es el empleo de internet relacionado con la asistencia a una consulta presen- cial con un profesional de la salud: en el presente estudio, las consultas en la red se llevaban a cabo antes 0 después de dicha consulta en un $63,9 \%$ de los casos, dato similar al de los internautas procedentes de la población general en el estudio de la ONTSI (65\%).

En el estudio de la ONTSI también se revelan algunos datos que nos ayudan en la integración de la información aquí analizada, como son los siguientes:

- Cuando se pregunta a la población general, solo 1 de cada 3 ciudadanos utiliza internet como fuente de información sobre temas de salud.

- Wikipedia (una enciclopedia virtual, en la que cualquiera puede añadir información sin un control profesional) es la web más utilizada para la búsqueda de información sobre salud.

- Solo el $12,7 \%$ de la población considera bastante 0 muy útiles los sistemas de salud basados en las TIC.

Los resultados obtenidos en la presente investigación también tienen parangón en trabajos internacionales; por ejemplo, con el hallazgo de que aproximadamente el $60 \%$ de los ciudadanos de la Unión Europea ha utilizado internet para buscar información de salud y que el $10 \%$ lo hace una vez o más por semana, o que el $62 \%$ lo hace principalmente para búsquedas relacionadas con modos de mejorar la salud (Fundación Telefónica, 2016), valores muy similares a los obtenidos en nuestro análisis. Otro estudio internacional que también arroja valores similares es el llevado a cabo en EE. UU. por Fontelo y Liu (2013), según el cual 1 de cada 3 propietarios de smarthpone utiliza sus terminales para hacer búsquedas sobre salud de todo tipo: textos informativos sobre temas de salud, diccionarios de enfermedades, control de hábitos (tabaco, alcohol, alimentación, sueño, conducta sexual, etc.), control o seguimiento de enfermedades o trastornos (diabetes, depresión, VIH, etc.), valoraciones del estado de salud, seguimiento de parámetros de salud, seguimiento del embarazo, etc.

\subsection{Propuestas de mejora en el empleo de la e-health}

Teniendo en cuenta las conclusiones previas, a continuación se sugieren unas líneas de actuación para acercar la e-health al ciudadano. 


\subsubsection{Mejorar las competencias digitales de la población}

Uno de los resultados que se han obtenido con esta investigación es que el medio más utilizado para buscar información sobre e-health son los buscadores tradicionales de internet, como Google. Sin embargo, estos presentan algunas singularidades que en el campo de la salud resultan significativas, por ejemplo, son buscadores generalistas y poco específicos, no establecen los resultados de las búsquedas en función de la calidad de la información a la que apuntan y, a veces, priorizan en los resultados información de fuentes que tienen intereses económicos. Si las anteriores matizaciones hacen que su uso deba ser cauto y crítico, en el campo de la salud deben aumentarse esas precauciones por las peligrosas consecuencias que podría conllevar seguir una indicación obtenida en una web para tomar decisiones sobre la propia salud.

La tradicional búsqueda de información en internet mediante buscadores genéricos debe ser una competencia digital que hay que depurar entre la población: el ciudadano no debe tener una actitud «inocente» cuando utiliza herramientas de empresas que tienen sus propios intereses a la hora de seleccionar los resultados y no se guían por criterios de interés público o ético.

\author{
Uno de los resultados que se han \\ obtenido con esta investigación \\ es que el medio más utilizado para \\ buscar información sobre e-health \\ son los buscadores tradicionales \\ de internet, como Google. [...] \\ La tradicional búsqueda de \\ información en internet mediante \\ buscadores genéricos debe ser una \\ competencia digital que hay que \\ depurar entre la población
}

\subsubsection{Mejorar la calidad de la información que está disponible en la red sobre la salud}

Dado el discreto resultado referido a la confianza que genera la información sobre la salud que puede obtenerse en internet y el empleo habitual de webs de escasa confianza, dicha herramienta podría tener mayor aval si se implementara algún sistema de evaluación y acreditación de la calidad, consideración que ya ha sido planteada previamente por otros autores (Conesa, Aguinaga y Hernández, 2011). Para ello podrían participar organismos públicos del ámbito de la salud y/o entidades profesionales de este ámbito asociadas a las diferentes temáticas o sistemas de distribución de la información. Así, cuando alguien hiciera una consulta o empleara alguno de los servicios en la red y observara el «sello» de acreditación, podría tener la tranquilidad de saber que los procesos de generación de la información, o la atención que allí pudiera obtener, son de confianza. Por ejemplo, ya que algunas de las aplicaciones más utilizadas están vinculadas al ejercicio y a la nutrición, el Ministerio de Salud, el Colegio de Médicos o incluso una asociación profesional independiente del ámbito de la nutrición podrían ser entidades candidatas
Dado el discreto resultado referido
a la confianza que genera la información sobre la salud que puede obtenerse en internet y el empleo habitual de webs de escasa confianza, dicha herramienta podría tener mayor aval si se implementara algún sistema de evaluación y acreditación de la calidad 
a ser organismos de acreditación. A este respecto no debemos olvidar que este tema de las acreditaciones y de los avales de entidades profesionales no está exento de polémica, sobre todo por la falta de regulación de estos procedimientos y el escaso consenso entre los especialistas y profesionales sobre qué, cómo y en función de qué acreditar. De hecho, recientemente, nutricionistas y dietistas criticaron el aval que ofrecía la Asociación Española de Pediatría a unas galletas para niños (mediante un sello que aparece en la caja junto a los datos nutricionales) para que las mismas sean consumidas en el desayuno, pues parece que contienen una cantidad excesiva de azúcar (Cadena SER, 2015).

Ya se han propuesto líneas concretas de trabajo en este campo, por ejemplo, el uso de varios sellos de calidad y códigos de conducta como los siguientes, que recoge GuíaSalud (2016): el uso de recomendaciones de la Unión Europea de 2002; contar con el HONcode de la Health on the Net Foundation (HON) 0
Fundación Salud en la Red, que es el sello de calidad más extendido actualmente a escala internacional; utilizar el código de conducta del programa de certificación de webs médicas, como la Web Médica Acreditada (WMA), que presenta el aval de un colegio profesional (el Colegio Oficial de Médicos de Barcelona) y es la que actualmente tiene mayor aceptación según una encuesta realizada por la HON; seguir los criterios del MedCIRCLE, un proyecto de la Unión Europea; o emplear el código ético y el sello de la Agencia de Calidad Sanitaria de Andalucía incluido dentro de su proceso de acreditación de páginas web sanitarias.

La anterior recomendación también podría aplicarse en el caso de las aplicaciones para dispositivos móviles, que en muchos casos les sirven a los ciudadanos para tomar decisiones sobre su salud, como la intensidad de la actividad física o el seguimiento de dietas, y que en bastantes ocasiones se emplean sin ningún fundamento científico e incluso con riesgo para la salud.

\subsubsection{Mejorar la formación y la educación: desarrollo de competencias}

Cuanta más formación posean los ciudadanos en aspectos relacionados con la salud, mayor capacidad tendrán para relacionarse de una forma eficaz y segura con la información y los servicios que ofrece la e-health, por ejemplo, a la hora de mantener una actitud crítica ante informaciones relativas a tratamientos, remedios o nutrición de dudosa consistencia. La educación para la salud debe ser responsabilidad de todos los sectores sociales (Administración, familias, empresas, etc.). Así, Pérez, Echauri, Ancizu y Chocarro (2006) han propuesto los siguientes ámbitos para llevar a cabo esta labor formativa: centros de educación formal (infantil, primaria, secundaria, postobligatoria, de adultos, etc.) y no formal (ocio y tiempo libre); servicios de salud (atención primaria de salud, centros de atención a la mujer, hospitales, otros centros y servicios de atención especializada, etc.); servicios municipales: sociales, de mujer, de juventud, socioculturales, etc.; servicios de salud laboral: servicios y delegados de prevención, empresarios, sindicatos, etc.; instancias político-administrativas: ayuntamientos, Gobierno y distintos departamentos, Parlamento, etc.; entidades sociales, organizaciones no gubernamentales (ONG), movimientos sociales, etc.; y canales de circulación cultural y estructuras sociales: grupos familiares, medios de comunicación social, grupos primarios y secundarios, etc.

\author{
Cuanta más formación posean \\ los ciudadanos en aspectos \\ relacionados con la salud, mayor \\ capacidad tendrán para relacionarse \\ de una forma eficaz y segura con \\ la información y los servicios \\ que ofrece la e-health [...] La \\ educación para la salud debe ser \\ responsabilidad de todos los sectores \\ sociales
}

En la actualidad ya nadie cuestiona que el mundo del siglo XXI será un mundo mediatizado por las TIC, por lo que es necesario asumir que unas adecuadas competencias digitales deben formar parte del currículo que todo ciudadano debe desarrollar. Según los resultados que se han obtenido en esta investigación es necesario mejorar las competencias digitales que permitan una mejor interacción del individuo con la e-health, algo que redundará en beneficio de todos. 


\section{Como objetivos que hay que desarrollar en lo que podríamos llamar una «alfabetización en e-healthm, planteamos los siguientes: saber encontrar los recursos de salud útiles, qué recursos de salud están disponibles, cómo encontrar recursos de salud útiles, cómo usar internet para preguntas de salud, cómo emplear la información de internet para encontrar ayuda, cómo confiar en la utilización de información para tomar decisiones respecto a la salud, saber distinguir entre recursos de alta o baja calidad y, por último, tener habilidades para evaluar los recursos de salud disponibles}

Como objetivos que hay que desarrollar en lo que podríamos llamar una «alfabetización en e-health», planteamos los siguientes, ya sugeridos por Rojas y Useche (2013): saber encontrar los recursos de salud útiles, qué recursos de salud están disponibles, cómo encontrar recursos de salud útiles, cómo usar internet para preguntas de salud, cómo emplear la información de internet para encontrar ayuda, cómo confiar en la utilización de información para tomar decisiones respecto a la salud, saber distinguir entre recursos de alta o baja calidad y, por último, tener habilidades para evaluar los recursos de salud disponibles.

A través de la concreción curricular, en las diferentes etapas educativas o en otros ámbitos de formación de

\section{BIBLIOGRAFÍA}

Cadena SER [2015]: Los nutricionistas cargan contra las galletas Dinosaurus. Disponible en: http://cadenaser. com/ser/2015/07/08/sociedad/1436370159_415853. html [Consultado: abril de 2016].

Conesa, M. ${ }^{a}$ del C.; Aguinaga, E. y Hernández, J. J. [2011]: «Evaluación de la calidad de las páginas web sanitarias mediante un cuestionario validado», Atención primaria, 43 (1), págs. 33-40. las competencias digitales, deberían trabajarse las siguientes competencias relacionadas con la búsqueda y gestión de la información en general, pero particularmente en e-health, que permitirian alcanzar los anteriores objetivos: ser capaz de realizar una navegación en internet a través de una búsqueda y selección crítica de información; conocer las estrategias que se utilizan en internet para publicar información, organizarla y realizar marcadores para que sea rastreada. También debería conocerse el papel de los negocios en internet a la hora de priorizar qué contenidos están ( 0 no) disponibles y accesibles; aprender a utilizar marcadores y alertas para clasificar y rastrear información relevante y de calidad; desarrollar estrategias de búsqueda en entornos específicos o utilizando buscadores específicos del ámbito de la salud: metabuscadores, buscadores profesionales o buscadores oficiales; ser capaz de desarrollar un programa de búsqueda personalizada o temática sobre aspectos específicos; establecer criterios y estrategias que permitan discriminar información fiable publicada en internet; aprender a diferenciar las fuentes de acreditación y validación de los contenidos de internet; saber qué posibles usos se pueden hacer de la información encontrada en la red; $y$ conocer las características de las aplicaciones de e-health: fundamento, objetivos, posibilidades, limitaciones, riesgos, etc.

La implantación de las pautas apuntadas en los apartados previos en los contextos señalados redundará en una visión más crítica y veraz de la e-health y en sus potenciales beneficios para la salud de los ciudadanos, quienes, a tenor de los resultados del estudio, están infrautilizando en la actualidad las TIC en este ámbito tan relevante para sus vidas, fundamentalmente por desconocimiento y desconfianza.

Eysenbach, G. [2001]: «What is e-health?», Journal of Medicine Internet Research, 3 (2): E20.

Fontelo, P. y Liu, F. [2013]: «Mobile app versus web app: a comparison using 2008-2012 "PubMed for Handhelds" server data», AMIA Annual Symposium Proceedings, 16, págs. 445-450.

Fundación Telefónica [2016]: La sociedad de la información en España 2015, Madrid: Ariel. 
GuíaSalud [2016]: Biblioteca de guías de práctica clínica en el Sistema Nacional de Salud (Calidad de la información de salud en la web/Recursos para pacientes/ Fuentes de información). Disponible en: http://portal. guiasalud.es/web/guest/calidad-informacion-salud [Consultado: abril de 2016].

Morales, M. [2015]: «España, a la cabeza del uso del móvil en Europa, y creciendo», Computerhoy.com. Disponible en: http://computerhoy.com/noticias/life/ espana-cabeza-del-uso-del-movil-europa-creciendo-28595 [Consultado: abril de 2016].

Oh, H.; Jadad, A.; Rizo, C.; Enkin, M.; Powell, J. y Pagliari, C. [2005]: «What is e-health (3): a systematic review of published definitions», Journal of Medicine Internet Research, 7 (1).
OMS [2005]: Cibersalud. Resolución WHA58.28. Disponible en: http://apps.who.int/iris/handle/10665/23104 [Consultado: abril de 2016].

ONTSI [2012]: Los ciudadanos ante la e-sanidad. Estudio sobre opiniones y expectativas de los ciudadanos sobre el uso y aplicación de las TIC en el ámbito sanitario, Ministerio de Industria, Energía y Turismo, Entidad Pública Empresarial Red.es.

Pérez, M.; Echauri, M.; Ancizu, E. y Chocarro, J. [2006]: Manual de educación para la salud, Pamplona: Gobierno de Navarra.

Rojas, D. F. y Useche, B. [2013]: «Alfabetización digital en salud: un análisis del constructo en la escala "eHealth Literacy Scale- eHeals" traducida al español», Revistaesalud.com, 9 (36).

\title{
ANEXO
}

\section{PROTOCOLO DE EVALUACIÓN DEL EMPLEO DELAS NUEVAS TECNOLOGIAS EN EL ÁMBITO DE LA SALUD}

\section{Instrucciones:}

- Esta encuesta es anónima.

- Te agradeceríamos que contestaras a las siguientes preguntas referidas al uso de las nuevas tecnologías en el ámbito de la salud. No te llevará más de 3 minutos.

- Si las opciones de respuesta se presentan con un círculo, solo podrás elegir una de las opciones. Si, por el contrario, se presentan con un cuadrado, podrás marcar tantas opciones como desees.

\section{Edad (indícala en número; por ejemplo, 37)}

\section{Género}

Hombre

$$
\text { Mujer }
$$

\section{3. ¿Qué titulación estás cursando actualmente en la UDIMA?}

\author{
Grado en Psicología \\ Grado en Magisterio de Educación Infantil \\ Grado en Magisterio de Educación Primaria \\ Grado en Derecho \\ Grado en Criminología \\ Grado en Ciencias del Trabajo, Relaciones Labores y Recursos Humanos \\ Máster en Psicología General Sanitaria
}




\section{PROTOCOLO DE EVALUACIÓN DEL EMPLEO DE LAS NUEVAS TECNOLOGIAS EN ELAMMBITO DE LA SALUD} .......

Máster en Gerontología Psicosocial

Máster en Dirección y Gestión de Centros Educativos

Máster en Formación del Profesorado de Educación Secundaria

\section{Señala tu nivel de formación, sin considerar los actuales estudios}

Has accedido a los estudios universitarios mediante la prueba de acceso a mayores de 25, 40045 años

Bachillerato

Diplomado, graduado o licenciado universitario

Máster

Doctorado

Otros

\section{Señala los dispositivos que utilizas habitualmente}

Ordenador personal de sobremesa

Ordenador portátil

Tableta

Teléfono móvil

\section{6. ¿Qué dispositivo sueles utilizar a la hora de realizar consultas en internet?}

Principalmente el teléfono móvil

Principalmente el ordenador o la tableta

Uso por igual el teléfono móvil y el ordenador o la tableta

7. ¿Utilizas alguno de estos dispositivos 0 aplicaciones de control y/o seguimiento de aspectos relacionados con la salud? Indica cuáles

Pulsera o dispositivo de control de actividad física

Aplicación en el móvil de seguimiento y/o control de la actividad física

Programa informático en el ordenador o en la tableta de seguimiento y/o control de la actividad física

Página web de seguimiento y/o control de la actividad física

No uso ninguno de los anteriores sistemas o dispositivos

\section{8. ¿Empleas alguna de las siguientes aplicaciones (app)? Indica cuáles}

Aplicación para el seguimiento y el control de la actividad física (control de pasos, gasto calórico, etc.)

Aplicación para el seguimiento y el control de la alimentación (número de calorías ingeridas, tipo de alimentos, etc.)

Aplicación para el seguimiento y el control de ambos: la actividad física y la alimentación

Otro tipo de aplicación referida a la salud

$\bigcirc$ Una aplicación que combina las anteriores

Ninguna aplicación de las mencionadas anteriormente 

......

9. ¿Realizas búsquedas de información sobre salud (información sobre enfermedades, tratamientos, síntomas, profesionales de la salud, etc.) en internet?
Sí
$\bigcirc$ No

10. Si realizas búsquedas de información sobre salud en internet, ¿con qué frecuencia lo haces?
A diario
Varias veces a la semana
Varias veces al mes
Una vez al mes o con menos frecuencia
Nunca realizo ese tipo de búsquedas

11. Si utilizas internet para buscar información sobre salud, ¿cómo lo sueles hacer?
Busco a través de buscadores, como Google
Consulto ciertas páginas concretas de salud
$\square$ Utilizo información disponible en redes sociales
$\square$ Utilizo otras fuentes de internet
No uso ningún sistema de los anteriores

12. ¿Qué grado de confianza tienes en la información que acerca de la salud puedes conseguir a través de internet? (1 implica una total desconfianza y 5 implica una total confianza)
$\bigcirc 1$
$\bigcirc 2$
$\bigcirc 3$
○
5

13. ¿Cuál de estas acciones llevas a cabo cuando acudes presencialmente a consulta por temas de salud (al médico de cabecera, al especialista, al psicólogo, al enfermero, etc.)?

Antes de ir, suelo consultar información en internet acerca de lo que me pasa

Después de la consulta (presencial), suelo consultar información en internet acerca de lo que me pasa y sobre lo que me ha dicho el especialista

Suelo consultar información antes y después de la consulta presencial

No suelo consultar información en internet ni antes ni después de la consulta presencial

14. ¿Cuál es el motivo para consultar información sobre temas de salud en internet?

$\square$ Tener más información sobre síntomas, enfermedades o tratamientos, aunque yo no tenga un problema de salud (ni nadie de mi círculo cercano)

$\square$ Intentar saber qué me pasa ante una duda de salud

$\square$ Tener más información sobre tratamientos de un posible problema de salud

Saber qué opinan otros usuarios de internet sobre un posible problema de salud o su tratamiento

Otros motivos/no hago consultas de este tipo

15. ¿Has seguido algún tratamiento relacionado con un problema de salud a partir de la información obtenida en internet?
Sí
No 


\section{PROTOCOLO DE EVALUACIÓN DEL EMPLEO DE LAS NUEVAS TECNOLOGIAS EN ELAMMBITO DE LA SALUD}

......

16. ¿Has iniciado un tratamiento farmacológico o has modificado uno ya recetado por un profesional médico a partir de la información obtenida en internet?
Sí
No

17. ¿Utilizas alguna de estas otras fuentes de información sobre salud?
Diccionarios médicos o de salud (en papel)
Libros especializados en salud (en papel)
Revistas médicas o especializadas en salud (en papel)
Ninguna de las anteriores

18. ¿Has consultado a profesionales de la salud a través de internet (psicólogos, nutricionistas, pediatras, ginecólogos, etc.)?
Sí
No

19. Si has contestado «sí» a la pregunta 18 , ¿en qué grado se resolvió tu duda o consulta? (1 significa que no se resolvió en absoluto y 5 significa que se resolvió totalmente)
$\bigcirc 1$
$\bigcirc 2$
3
$\bigcirc$
5

20. Si has contestado «no» a la pregunta 18 , ¿consultarías a un profesional de la salud a través de internet?
Sí
No
Contesté «sí» a la pregunta anterior

21. ¿Has recibido tratamiento online por parte de un profesional de la salud?
Sí
No

22. Si has contestado «sí») a la pregunta 21 , ¿cómo valoras el resultado? (1 significa muy malo, insatisfactorio; 5 significa muy bueno, satisfactorio)
$\bigcirc 1$
2
3
$\bigcirc$
5

23. ¿Cuál es tu grado de confianza en un servicio de salud proporcionado a través de internet? (1 implica muy poca confianza y 5 implica total confianza)
$\bigcirc 1$
$\bigcirc 2$
3
$\bigcirc 4$
5

24. En comparación con el servicio presencial (cara a cara) relacionado con temas de salud, ¿cuál es tu valoración del servicio online?

Considero el servicio presencial mejor que el servicio online

Considero el servicio presencial igual que el servicio online

Considero el servicio presencial peor que el servicio online 


\section{PROTOCOLO DE EVALUACIÓN DEL EMPLEO DE LAS NUEVAS TECNOLOGIAS EN EL ÁMBITO DE LA SALUD} $\ldots / \ldots$

\section{Si no emplearas servicios de salud online, ¿a qué se debería?}

\section{$\square$ Motivos económicos}

Desconfianza en la identidad del profesional

$\square$ Desconfianza en la profesionalidad del profesional

Posibles contratiempos técnicos

$\square$ Otros

La másteres oficiales que ofrece la UDIMA tienen como señas de identidad la cercanía del profesor al alumno y la mejor aplicación posible de las TIC a la formación. La UDIMA es una institución de enseñanza pensada y diseñada para las personas que trabajan y para quienes por motivos de horarios, movilidad, distancia geográfica o de conciliación familiar demandan una universidad abierta y flexible. Este máster oficial [60 créditos ECTS] se inicia en octubre y febrero de cada año y su duración normal es de 12 meses.

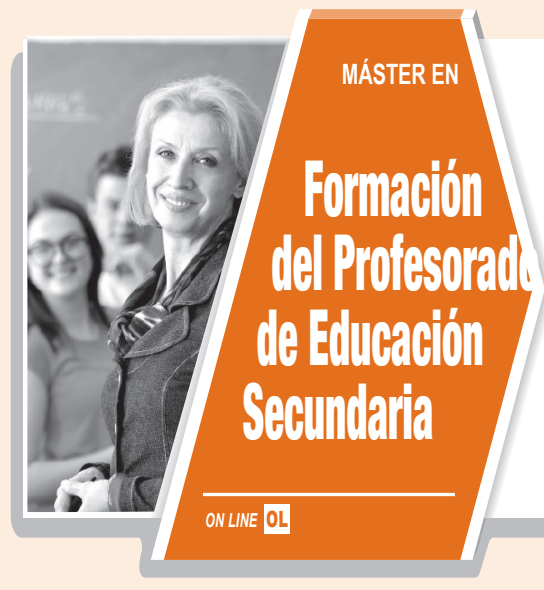

DIRIGIDO A: La Orden ECI/3858/2007 establece que los alumnos que deseen acceder a este máster, además de los requisitos generales, deben cumplir: acreditación del dominio de las competencias relativas a la especialización que se desee cursar y dominio de una lengua extranjera equivalente al nivel B1 del Marco Común Europeo de Referencia para las lenguas.

OBJETIVOS: Adquirir las competencias necesarias para poder ejercer la profesión docente en Secundaria, Bachillerato, Formación Profesional y enseñanzas de idiomas de acuerdo con la normativa vigente. 